\title{
Remote Monitoring of Heading Rice Growing and Nitrogen Content Based on UAV Images
}

\author{
Yu Zhang, Zhongbin Su*, Weizheng Shen, Renshan Jia, Jiling Luan \\ College of Electrical and Information, Northeast Agricultural University, \\ Harbin 150030, China \\ yubuting619@gmail.com
}

\begin{abstract}
Rice heading is the critical stage of the growth of rice, rice plants are relatively large, field canopy, poor resistance, and coincided with the high temperature and rainy weather, pests and diseases are more prone period. Therefore, the use of UAV to monitor the growth status of rice, easy to understand rice growth and nutritional status, in order to achieve high-quality, high yield, efficient purpose. This article is the field experiments under different nitrogen levels, using UAV monitoring multispectral images of rice, by reference remote sensing spectral indices, derived green normalized difference vegetation index (GNDVI) relative to other spectral index is more suitable for rice field biomass inversion modeling. At the same time, this study is based on the inversion model, implemented the rice growing and nitrogen content graded by ISODATA methods in ENVI. Achieved classification on rice growth and nitrogen remote sensing thematic map in ArcGIS, to provide timely and accurate information for rice seedling diagnosis and management decision, has reached the purpose of rice production precise management.
\end{abstract}

Keywords: rice, UAV image, GNDVI, growth monitoring, nitrogen content classification

\section{Introduction}

In recent years, precision agriculture has become the most attractive field of cutting-edge international hotspot which can reasonably use of agricultural resources and improving the quality of agricultural products. Using UAV remote sensing information platform to obtain information on field crops, with low running costs, high flexibility, access to data fast in real-time and other characteristics. It is one of the main methods for the rapid acquisition of agricultural crop information. And it is an important direction of development of precision agriculture.

Research on remote sensing of rice growing, most of the formers are based on the analysis of the relationship between the spectral information of remote sensing images (such as NDVI, RVI, etc.) with the LAI or biomass of rice through the establishment linear regression mode to quantitative analysis of rice growing[1-5]. Such as the use of TM's NDVI vegetation index image inversion leaf area index of growth monitoring method[6]; the use of vegetation index RVI inversion growing biomass monitoring method[7]; the use of MODIS data acquisition rice parameter information, obtain information through the establishment of rice growing method of nonlinear model[8]. In recent years, vegetation index and SPAD value have been widely used in crop nutrition diagnosis[9-14].

In this paper, the change of canopy vegetation index of rice under different nitrogen fertilization levels was studied by using the UAV image data of rice heading stage. And analyze the relationship between vegetation index and SPAD value of leaves and leaf 
nitrogen content. Data research shows that the vegetation index GNDVI is the best choice for the diagnosis of rice biomass and nutritional status, which provides a theoretical basis for guiding the precision of nitrogen fertilizer. Coupled with extraction of rice area based on ISODATA classification method[15], carried out rice plant growing and nitrogen contend monitoring by UAV. The production of remote sensing thematic maps for rice growth and nitrogen classification was achieved. For basic agricultural technical personnel, the remote sensing thematic maps can easy understand and application. The remote sensing thematic maps also can provide timely and accurate information for rice seedling diagnosis and management decision, has reached the purpose of rice production precise management.

\section{Overview of Research Area}

Founder county is located in the middle east of Harbin city, Heilongjiang province, a population of about 212300. The total area of 2993 square kilometers, the grain crops sown area of 73619 hectares. And Founder county belongs to the hills and low mountain terrain, the elevation is generally between 300 to 700 meters. The climate is a temperate continental monsoon climate, precipitation is rich, the average annual rainfall of $579.7 \mathrm{~mm}$, it is a mid-latitude region. The growth period of the field crops is May-September. The total sunshine hours are 1178 hours, the sunshine percentage is $54 \%$, and the average is 8 hours per day.

The airborne region to the Founder Rice Research Institute as a starting point, measuring field block neat, and provide convenience for remote sensing image classification. At the same time, ground on crop growth monitoring is adequate, convenient access to information on a variety of crops, provides reliable guarantee to UAV image of agricultural monitoring application research.

\section{Materials and Methods}

\subsection{Field Experiment Design}

This experiment was conducted in the Founder Rice Research Institute, with $\mathrm{Wu}$ Youdao 3 and Yang Guang 4 as experimental research object, which is divided into north and south two areas. According to the distribution of rice planting in experimental field, 6 fertilizer gradients are set up: N0 (nitrogen-free), N1 $\left(60 \mathrm{~kg} / \mathrm{hm}^{2}\right), \mathrm{N} 2$ $\left(90 \mathrm{~kg} / \mathrm{hm}^{2}\right)$, N3 (Pure nitrogen $\left.120 \mathrm{~kg} / \mathrm{hm}^{2}\right), \mathrm{N} 4\left(150 \mathrm{~kg} / \mathrm{hm}^{2}\right), \mathrm{N} 5\left(180 \mathrm{~kg} / \mathrm{hm}^{2}\right)$. For serious lack of nitrogen, nitrogen deficiency, the amount of nitrogen, excess nitrogen and other conditions, respectively, fertilizer in basal fertilizer was applied $40 \%$, reviving stage (3-5 days after transplanting) was applied $40 \%$, and the jointing stage (main spike length of $5 \mathrm{~mm}$ ) was applied $30 \%$. In the base fertilizer and the jointing stage, twice a uniform amount of potash fertilizer was applied in each test plot, the potassium chloride $3 \mathrm{bags} / \mathrm{hm}^{2}$, calcium superphosphate $2 \mathrm{bags} / \mathrm{hm}^{2}$. Each level has 4 repetitions, a total of 24 test plots. The specific distribution is shown in Table 1.

Table 1. Rice Field Test Plot Distribution Table

\begin{tabular}{llll}
\hline \multicolumn{4}{c}{ Nitrogen content of rice experimental area } \\
\hline N2 & N0 & N3 & N5 \\
N3 & N1 & N4 & N0 \\
N4 & N2 & N5 & N1 \\
N5 & N3 & N0 & N2 \\
N0 & N4 & N1 & N3 \\
N1 & N5 & N2 & N4 \\
\hline
\end{tabular}




\subsection{Remote Sensing Data Acquisition and Preprocessing of UAV}

This experiment uses a fixed-wing UAV TY-06, the UAV can load $8 \mathrm{~kg}$, flight date is July 27 to 30 (rice heading stage). The flight time is $30 \mathrm{~min}$, flying height is $550 \mathrm{~m}$, and flying area is $666.7 \mathrm{hm} 2$. The UAV equipped with high-definition digital camera (SONY 5100, resolution is 6000*4000) and multi-spectral camera (ADC Lite Tetracam, include near-infrared band, red band, and green band, resolution is 2048*1536) to obtain orthophotos and canopy spectral information in rice growing areas. Aerial photography included experimental plots of north and south. The specific parameters are shown in Table 2.

Table 2. UAV Aerial Parameters Table

\begin{tabular}{cc}
\hline \multicolumn{2}{c}{ UAV aerial parameters } \\
\hline Flight altitude $(\mathrm{m})$ & 550 \\
Ground resolution $(\mathrm{m})$ & 0.2199 \\
Accuracy & $1 / 3000$ \\
Ground range $(\mathrm{m})$ & $337 * 450$ \\
$72 \%$ course overlap baseline length $(\mathrm{m})$ & 126 \\
$44 \%$ off the pitch to overlapping routes $(\mathrm{m})$ & 189 \\
\hline
\end{tabular}

Each flight of UAV shot 319 images, aerial point as shown in Figure 1. Select the good effect of high-definition digital images, using Agisoft Photoscan software to quickly mosaic, to generate a UAV HD image of Founder Rice Research Institute, which coverage of the research base of rice and residential areas. After geometric correction of ADC multi-spectral image, used Pix4Dmapper software to mosaic image to generate multi-spectral image. And ADC multi-spectral image to generate a multi-spectral reflectance image by the strict radiometric calibration. Taking into account the impact of light time difference on the multi-spectral images, using pseudo-standard feature radiometric calibration method to measuring the reflectivity of the cloth surface so that multi-spectral image was corrected. After treatment, the multi-spectral image has a more real reflectivity.

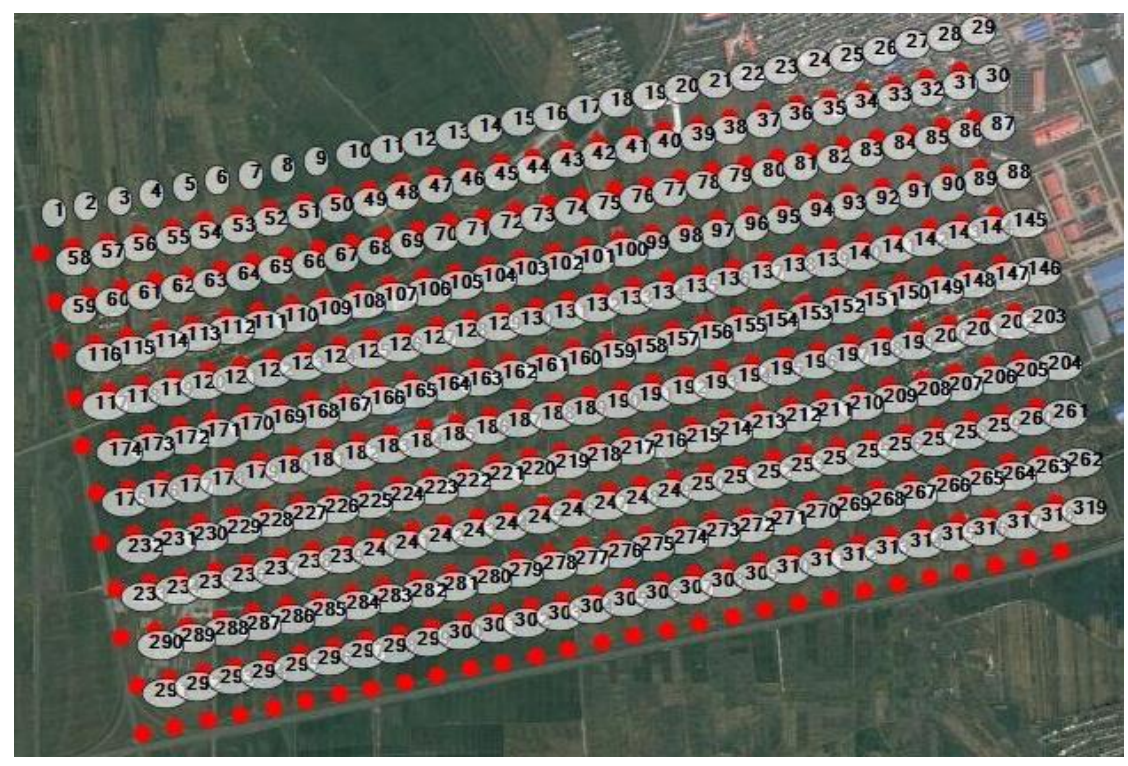

Figure 1. UAV Aerial Point Figure 


\subsection{Ground Data Collection and Thematic Map Production}

The ground observation point is the use of plant nutrient analyzer TYS-4N and millimeter ruler to collect the SPAD values, nitrogen content of rice canopy and plant height. Each test plot collected 10 samples. A total of 480 samples used for establishing biomass inversion model. In the non-experimental area, 60 samples were selected for the detection of the model. Varieties of these samples are NEAU 426.

Multi-spectral images after pretreatment with Band Math of ENVI software, draw relevant vegetation index image. And correlation analysis combined with ground samples measured data and its vegetation index. According to the analysis results, rice growth and nitrogen classification were carried out. The last achieved classification on rice growth and nitrogen remote sensing thematic map in ArcGIS. Specific processes are shown in Figure 2.

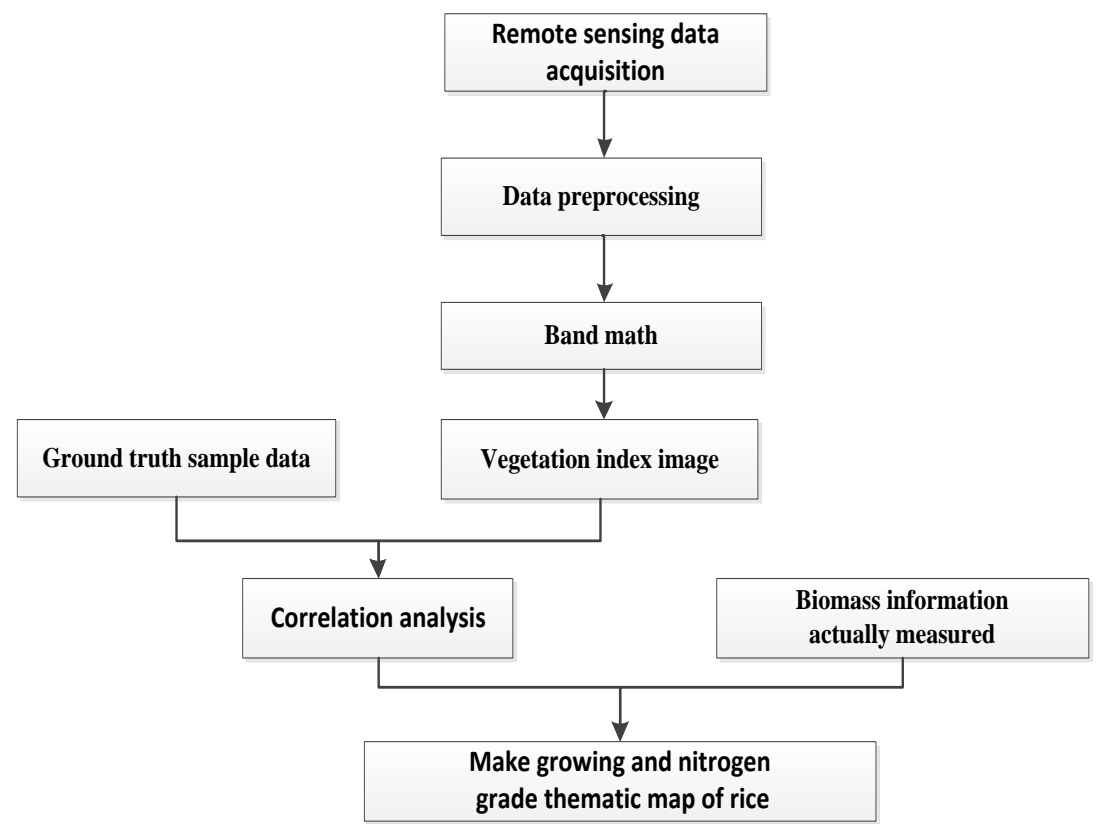

Figure 2. Classification Flow Chart of Remote Sensing Monitoring of Rice Growth and Nitrogen Content

\section{Data Extraction and Analysis}

\subsection{The UAV aerial data analysis}

Remote sensing spectral index, which can weaken the interference of background information on spectral characteristics of vegetation by linear or non-linear combination of different wave bands, can improve the accuracy of remote sensing data in the expression of chlorophyll and nitrogen content[16-19]. In this paper, the remote sensing spectral indexes are shown in Table 3 . The vegetation index value corresponding to each sampling point was extracted from the processed remote sensing image, which can establish the regression model of SPAD value and nitrogen content of rice canopy. And find out the vegetation index which is suitable for the build of rice growing and nitrogen inversion model. 
Table 3. The Remote Sensing Spectral Indexes Selected in this Research

\begin{tabular}{ll}
\hline \multicolumn{1}{c}{ Remote sensing spectral index } & \multicolumn{1}{c}{ Calculation formulas } \\
\hline Difference vegetation index(DVI) & DVI=B1-B2 \\
Ratio vegetation index(RVI) & RVI=B1/B2 \\
Normalized difference vegetation index(NDVI) & NDVI=(B1-B2)/(B1+B2) \\
Green normalized difference vegetation index(GNDVI) & GNDVI=(B1-B3)/(B1+B3) \\
Soil adjusted vegetation index(SAVI) & SAVI=(B1-B2)/(B1+B2+0.5)*(1+0.5) \\
Optimal soil adjusted vegetation index(OSAVI) & OSAVI=1.16*(B1-B2)/(B1+B2+0.16) \\
\hline
\end{tabular}

Note: B1, B2 and B3 denoted spectrum reflectance of ADC multispectral images at near infrared, red and green bands, respectively. The same below.

\subsection{Analysis of Ground Measured Point Data}

Plant nitrogen content and chlorophyll are the important nutrition and physiological parameters of plant growth, which is an important parameter to reflect the vital signs. And it is also an important basis for plant fertilization and irrigation. The results of the determination of chlorophyll content in plant nutrient analyzer TYS-4N were SPAD values. And SPAD values are often indirectly representative of chlorophyll values. So it can be used to assess the health status and the growth status of plants. Thus, TYS-4N often used to detect the chlorophyll content of plants, in order to guide the amount of nitrogen fertilizer in agriculture.

In each block of test plots selected 10 rice plants growing evenly, with plant nutrient analyzer TYS-4N measured SPAD value and nitrogen content of the top 1 leaf. Then, the mean value of SPAD value and nitrogen content of the test plots were used as the SPAD value and nitrogen content of rice canopy under different nitrogen. The processed data are shown in Table 4 and Table 5.

Table 4. SPAD Values in the Rice Canopy Under Six Levels of Nitrogen Fertilization

\begin{tabular}{ccccccc}
\hline \multirow{2}{*}{ Rice varieties (A) } & \multicolumn{7}{c}{ Nitrogen levels (B) } \\
\cline { 2 - 7 } & $\mathrm{N} 0$ & $\mathrm{~N} 1$ & $\mathrm{~N} 2$ & $\mathrm{~N} 3$ & $\mathrm{~N} 4$ & $\mathrm{~N} 5$ \\
& $(\mathrm{~B} 1)$ & $(\mathrm{B} 2)$ & $(\mathrm{B} 3)$ & $(\mathrm{B} 4)$ & $(\mathrm{B} 5)$ & $(\mathrm{B} 6)$ \\
\hline \multirow{2}{*}{ Wu Youdao 3 } & 26.82 & 31.52 & 34.2 & 34.49 & 37.76 & 39.36 \\
(A1) & 24.31 & 31.64 & 34.69 & 35.37 & 36.51 & 37.39 \\
& 23.38 & 30.32 & 30.87 & 32.85 & 33.4 & 34.18 \\
& 24.82 & 27.95 & 29.45 & 31.02 & 32.12 & 33.08 \\
\hline \multirow{2}{*}{ Yang Guang 4 } & 26.72 & 28.7 & 31.61 & 36.01 & 38.83 & 39.97 \\
(A2) & 34.12 & 35.84 & 36.98 & 38.34 & 38.48 & 42.57 \\
& 23.17 & 31.46 & 34.17 & 37.41 & 37.93 & 39.37 \\
& 24.94 & 34.98 & 35.62 & 36.03 & 36.49 & 36.81 \\
\hline
\end{tabular}

Table 5. Nitrogen Contents in the Rice Canopy under Six Levels of Nitrogen Fertilization

\begin{tabular}{ccccccc}
\hline \multirow{2}{*}{ Rice varieties (A) } & \multicolumn{7}{c}{ Nitrogen levels (B) } \\
\cline { 2 - 7 } & N0 & N1 & N2 & N3 & N4 & N5 \\
& $(B 1)$ & $(B 2)$ & (B3) & (B4) & (B5) & (B6) \\
\hline \multirow{2}{*}{ Wu Youdao 3 } & 2.43 & 2.78 & 2.96 & 2.98 & 3.21 & 3.32 \\
(A1) & 2.27 & 2.78 & 3.00 & 3.03 & 3.13 & 3.18 \\
& 2.19 & 2.68 & 2.73 & 2.84 & 2.9 & 2.95 \\
& 2.28 & 2.51 & 2.62 & 2.73 & 2.82 & 2.89 \\
\hline
\end{tabular}




\begin{tabular}{cllllll} 
Yang Guang 4 & 2.96 & 3.08 & 3.15 & 3.24 & 3.26 & 3.57 \\
(A2) & 2.17 & 2.76 & 2.96 & 3.17 & 3.23 & 3.32 \\
& 2.30 & 3.00 & 3.05 & 3.08 & 3.11 & 3.14 \\
\hline
\end{tabular}

Analysis can be made from Table 4 and Table 5 that the SPAD value was proportional to the nitrogen content of the rice canopy and unaffected by rice varieties. Its linear regression model is shown in Figure 3. So that the nitrogen content of rice canopy can be obtained by the SPAD value.

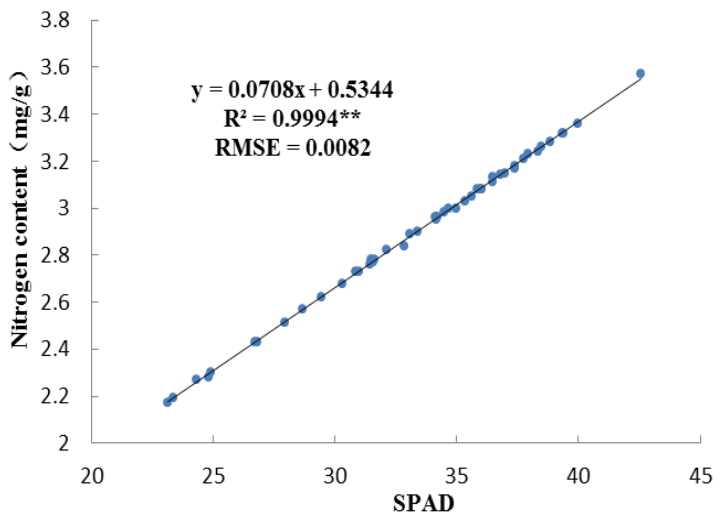

Figure 3. Correlation between SPAD and Nitrogen Content of Rice Canopy in Heading Stage

Note: ** indicated significant correlation at the 0.01 level. The same below.

Through the variance analysis in Table 4 that the results were shown in Table 6.

Table 6. Analysis of Variance Table

\begin{tabular}{ccccccc}
\hline Source of variation & Freedom & quadratic sum & Mean square & $F$ & F 0.05 & F 0.01 \\
\hline A treatment & 1 & 99.33 & 99.33 & 15.34 & 4.11 & 7.4 \\
B treatment & 5 & 718.66 & 143.73 & 22.19 & 2.48 & 3.57 \\
A $\times$ B & 3 & 3.73 & 1.24 & 0.19 & 2.87 & 4.38 \\
Error & 36 & 233.16 & 6.48 & & & \\
Total variation & 47 & 1054.87 & & & & \\
\hline
\end{tabular}

Analysis critical F value of table 6: F A > F 0.01 (1,36) =7.4, F B > F 0.01 $(5,36)$ $=3.57, \quad \mathrm{FA} \times \mathrm{B}<\mathrm{F} 0.05(3,36)=4.38$. We can know that the rice varieties had a significantly effect on the SPAD , and the level of nitrogen application had a significant effect on SPAD, and the interaction between rice varieties and nitrogen application had no significant effect on SPAD of rice canopy during heading. The SPAD value of rice canopy was proportional to its level of nitrogen application. Rice test plots with severe nitrogen deficiency and excess nitrogen fertilizer could be distinguished by SPAD classification of rice canopy.

\subsection{Correlation between SPAD Value and Vegetation Index of Rice Canopy}

By analysis the correlation between vegetation index and ground measured data, obtained a linear regression mode shown in Table 7. As long as the correlation between vegetation index and SPAD value of canopy leaves was established, the content of nitrogen in the canopy leaf can be obtained, the growth and nitrogen of rice plants can be evaluated. So as to further clarify the nutrition level of rice plant. 
Table 7. SPAD and Vegetation Index Regression Model of Rice Canopy

\begin{tabular}{cllll}
\hline \multirow{2}{*}{$\begin{array}{c}\text { vegetation } \\
\text { index }\end{array}$} & \multicolumn{2}{c}{ Wu Youdao 3} & \multicolumn{2}{c}{ Yang Guang 4} \\
\cline { 2 - 5 } & \multicolumn{1}{c}{ Linear regression model } & \multicolumn{1}{c}{$\mathrm{R}^{2}$} & \multicolumn{1}{c}{ Linear regression model } & $\mathrm{R}^{2}$ \\
\hline DVI & $\mathrm{y}=0.7728 \mathrm{x}+6.5213$ & $0.8656^{* *}$ & $\mathrm{y}=0.754 \mathrm{x}+5.0631$ & $0.7159^{* *}$ \\
RVI & $\mathrm{y}=2.7441 \mathrm{x}+13.525$ & $0.8619 * *$ & $\mathrm{y}=1.7271 \mathrm{x}+18.952$ & $0.6508^{* *}$ \\
NDVI & $\mathrm{y}=74.434 \mathrm{x}-22.399$ & $0.855^{* *}$ & $\mathrm{y}=90.649 \mathrm{x}-36.963$ & $0.7401 * *$ \\
GNDVI & $\mathrm{y}=153.25 \mathrm{x}-16.86$ & $0.9478^{* *}$ & $\mathrm{y}=171.68 \mathrm{x}-23.917$ & $0.8587 * *$ \\
SAVI & $\mathrm{y}=51.153 \mathrm{x}-23.041$ & $0.8694 * *$ & $\mathrm{y}=60.201 \mathrm{x}-35.706$ & $0.7715^{* *}$ \\
OSAVI & $\mathrm{y}=66.381 \mathrm{x}-23.991$ & $0.885^{* *}$ & $\mathrm{y}=78.454 \mathrm{x}-36.89$ & $0.7899^{* *}$ \\
\hline
\end{tabular}

From Table 7, the correlation of GNDVI with SPAD value of rice canopy was higher than other vegetation index. Mixing two kinds of rice samples to obtained linear regression models as shown in Figure4. And GNDVI is more suitable for field crops inversion biomass we found.
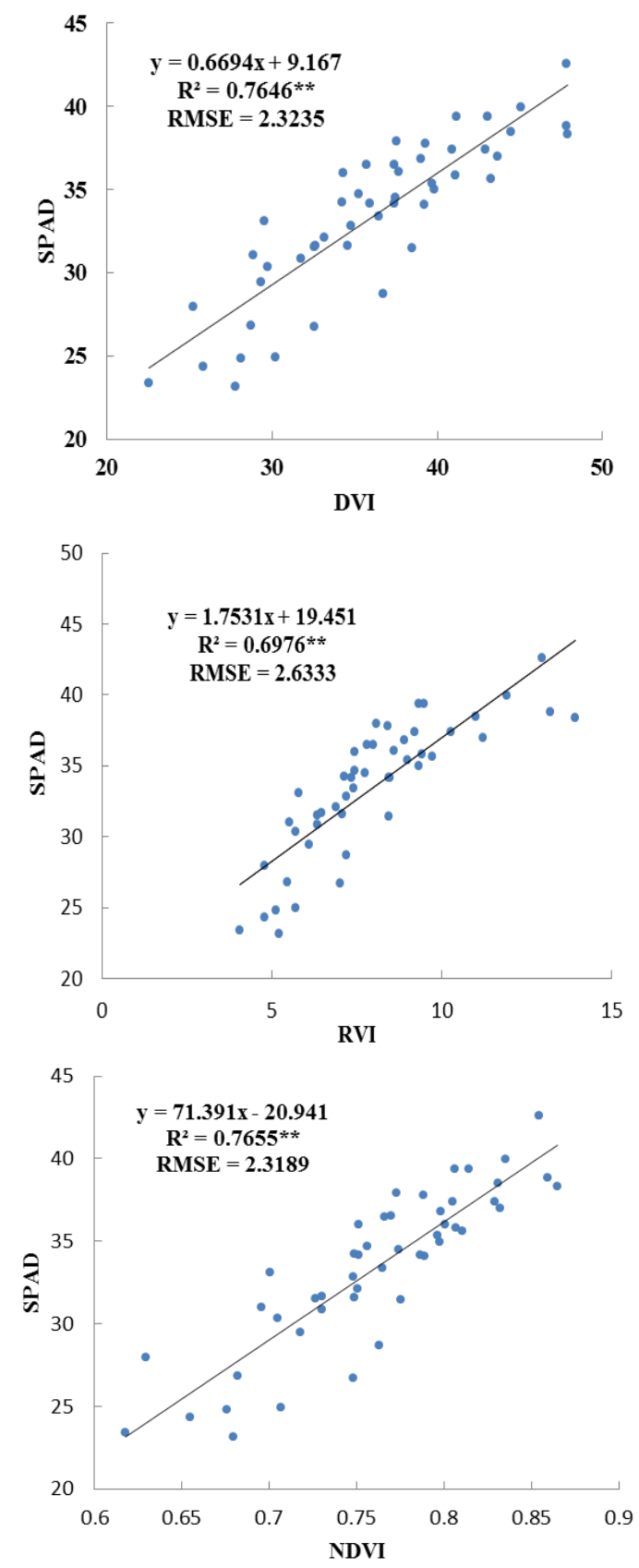

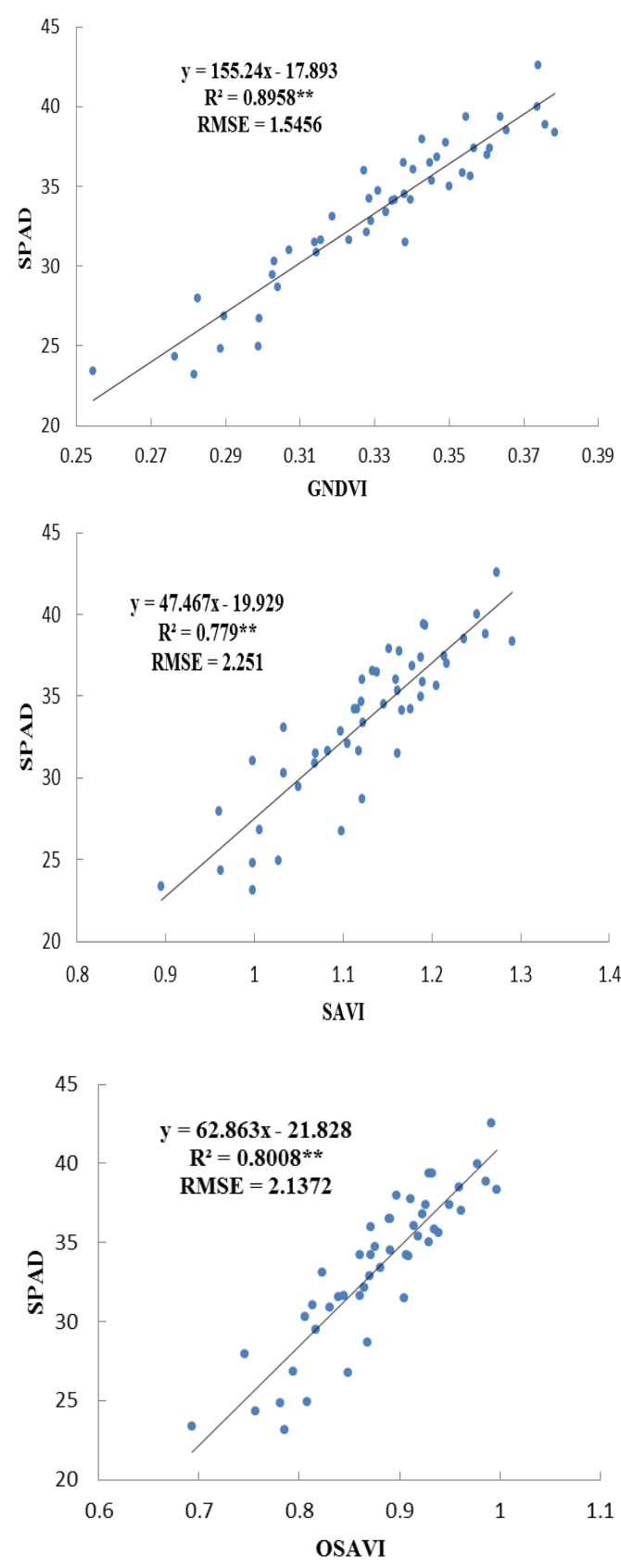

\section{Figure 4. Build SPAD and Vegetation Indexes Inversion Model in Rice Heading Stage}

The inversion model of SPAD was used to carry out the Dong Nong 426 samples, and comparison with the actual measured SPAD values. Establishment and verification of GNDVI and SPAD inversion model as shown in Figure 5 where $\mathrm{N}$ is the number of samples. The analysis shows that the green normalized difference vegetation index (GNDVI) can be used as a sensitive variable for the inversion of SPAD and nitrogen content of rice, and the constructed remote sensing inversion model is reliable and has higher precision. 


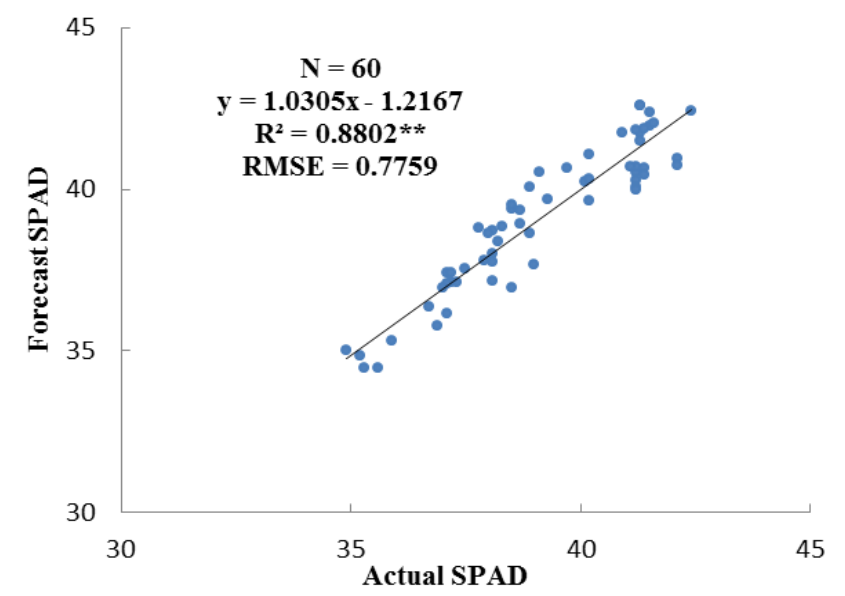

\section{Figure 5. Validation of SPAD and GNDVI Inversion Model in Rice Heading Stage}

\subsection{Application}

In heading stage, the green normalized difference vegetation index (GNDVI) can be used as a sensitive variable for the inversion of SPAD and nitrogen content of rice. And the remote sensing inversion model is reliable which has higher precision. On this basis, using ISODATA classification method to obtain the classification image of SPAD and nitrogen content. Based on the data analysis, the growth of rice was divided into 4 grades, and nitrogen content was divided into 3 grades in the rice heading stage of Founder county. The remote sensing classification map of rice growth and nitrogen was made in ArcGIS, which provides a fast and convenient seedling parameters extraction method of large area crop, as shown in Figure 6 and Figure 7.

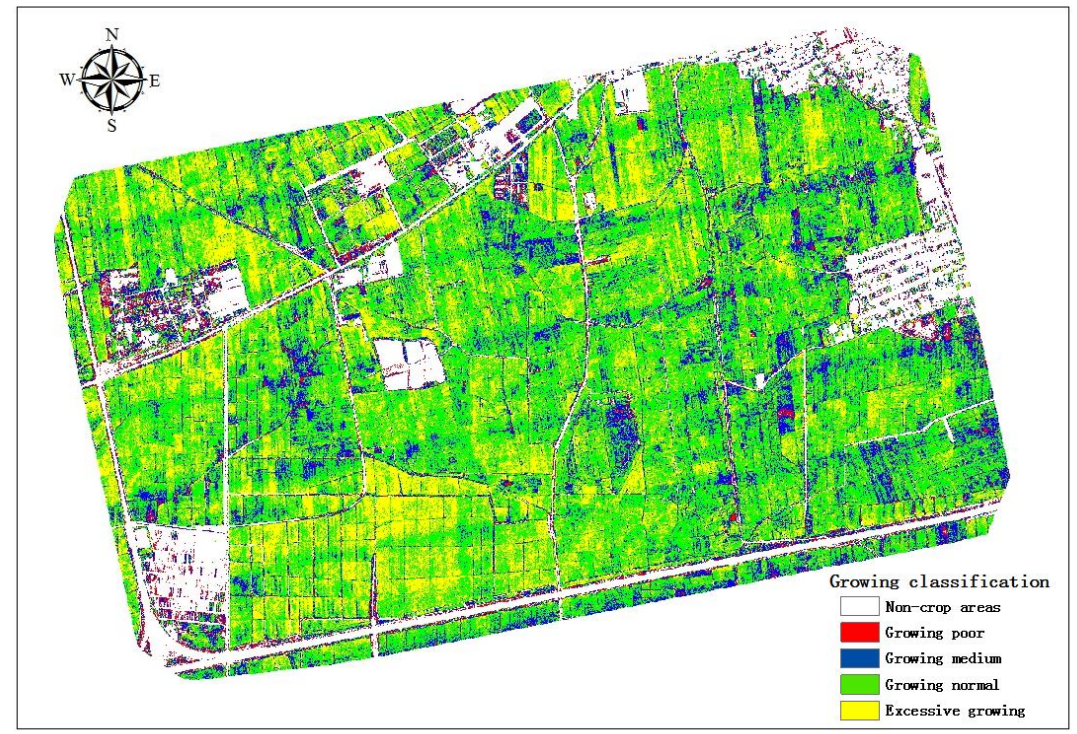

\section{Figure 6. Rice Growth Monitoring by Remote Sensing Classification Map in Heading Stage}

Figure 6 shows that SPAD value of rice canopy which less than 28 is growing poor, its area accounted for aerial monitoring rice area's $3.053 \%$, about $331804.782 \mathrm{~m}^{2}$. The SPAD value between 28 and 32 is growing medium, its area accounted for $10.291 \%$ of the aerial monitoring area, about $1118281.3129 \mathrm{~m}^{2}$. The value of SPAD between 32 and 36 is normal growth, which area accounted for $33.071 \%$ of the aerial monitoring rice 
area, about $3593688.7070 \mathrm{~m}^{2}$. And SPAD value is greater than 36 is the growth of excess, its area accounted for $17.805 \%$ of the aerial monitoring area, about $1934823.6158 \mathrm{~m}^{2}$.

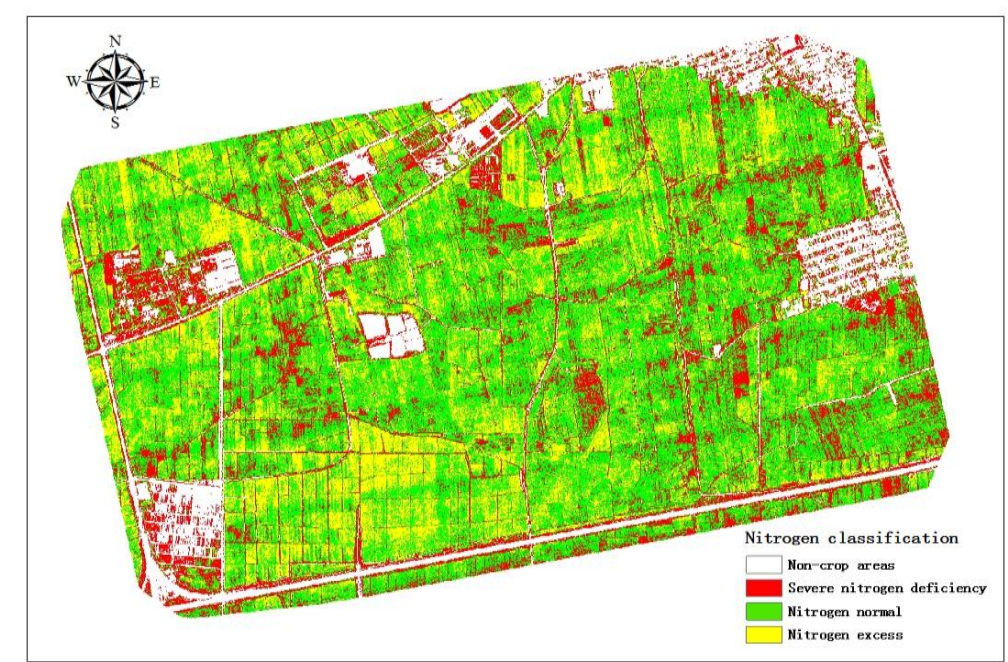

\section{Figure 7. Rice Nitrogen Monitoring by Remote Sensing Classification Map in Heading Stage}

Figure 7 shows that Nitrogen content $>3.2 \mathrm{mg} / \mathrm{g}$ represent nitrogen excess, its area accounted for aerial monitoring area's $17.805 \%$, about $1934823.6158 \mathrm{~m}^{2}$. The nitrogen content $<2.7 \mathrm{mg} / \mathrm{g}$ represent serious lack of nitrogen, its area accounted for $15.036 \%$ of the aerial monitoring area, about $1633970.3887 \mathrm{~m}^{2}$. And normal nitrogen content in the $2.7-3.2 \mathrm{mg} / \mathrm{g}$, its area accounted for aerial monitoring area's $33.071 \%$, about $3593688.7070 \mathrm{~m}^{2}$. Overall analysis, the Founder county's rice growing well this year. Grade has differences explain that some problems exist in the management of rice field, still has the potential to be tapped. Such as rice field with poor growth or low nitrogen, should apply appropriate amount of panicle fertilizer to promote the growth of pollen and flower to increase production.

In this paper, the production remote sensing thematic maps of rice growth and nitrogen can help agricultural technical personnel to guide the field production management which has a good practical. In order to facilitate the work of agricultural technical personnel, the boundary vector map of each township can be superimposed on the remote sensing thematic maps, which can more accurately understand the township rice distribution area, growing and nitrogen situation, so as to formulate appropriate field management measures to achieve the purpose of increasing and stabilizing the yield. In addition, it can also be superimposed by remote sensing thematic maps and vector image of land ownership in rural areas, so as to guide the farmers proper fertilization according to their own rice growth and nitrogen content classification map, to achieve precision agriculture, as shown in Figure 8. 


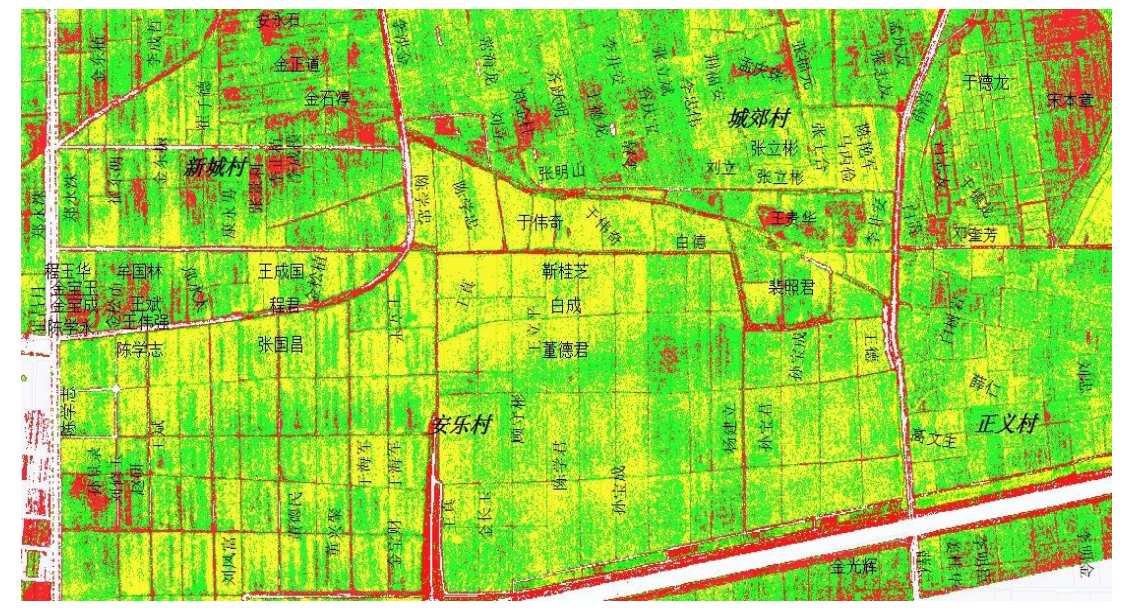

\section{Figure 8. The Practical Application of Remote Sensing Classification Map of Rice's Nitrogen Content in Heading Stage}

\section{Discussion}

In this study, the relationship between the vegetation index, SPAD value and nitrogen content which under different nitrogen levels in rice heading stage were fitted by linear regression. Among them, for a single species, the relationship between GNDVI and SPAD value is the most closely, and its model accuracy is very high. And for multiple varieties of rice build inversion model, its $\mathrm{R}^{2}$ reached 0.8958 . It is proved that the inversion of rice growth and nitrogen content by GNDVI is feasible. Because of the difference of GNDVI among different rice varieties, it also needs a lot of work to increase the inversion model's universality and accuracy. In addition, this study only carried out a year of fixed point test, the results of the actual application needs to be further verified. The method of this paper can only obtain the instantaneous growth and nitrogen content information of rice. To carry out dynamic monitoring the growth and nitrogen content of rice, we need to use multi-temporal remote sensing image data or to establish a dynamic monitoring model of rice growing and nitrogen content.

\section{Acknowledgements}

The authors would like to acknowledge the financial support from the National High-tech R\&D Program of China (863 Program) (2013AA102303) and Key Technologies R\&D Program of Heilongjiang Province (GA15B106, GA15B107).

\section{References}

[1] Weiguo Li, Bingbai Li and Chunlin Shi. Research Progress in Rice Condition Monitoring Based on Growth Model and Remote Sensing[J]. Chinese Agricultural Science Bulletin. 22(9), 457-461(2006).

[2] Hongwei $\mathrm{Xu}, \mathrm{Ke}$ Wang. Regionalization for Rice Yield Estimation by Remote Sensing in Zhejiang Province[J]. Pedosphere. 11(2), 175-184(2001).

[3] Rui Zhao, Junyou Tang and Longhua He. Study on Optimized Patterns of Soil and Water Conservation Measures on Sloping Fields in Rocky Mountainous Area of Northern China[J]. Remote Sensing For Land \& Resources. 53(3), 9-11(2002).

[4] Qian Cheng. Models for rice yield estimation using remote sensing data of MOD13[J]. Transactions of the Chinese Society of Agricultural Engineering. 22(3), 79-83(2006).

[5] ABOU-ISMAIL O, Jingfeng Huang and Renchao Wan. Rice Yield Estimation by Integrating Remote Sensing with Rice Growth Simulation Model[J]. Pedosphere. 14(4), 519-526(2004).

[6] Weiguo Li, Jihua Wang, Chunjiang Zhao, Qingxi Tong and Liangyun Liu. Study on Monitoring the Winter Wheat Growth and Nitrogen Based on Landsat TM Image[J]. Remote Sensing Information. (2), 12-15(2007). 
[7] Weiguo Li, Jihua Wang, Cunjun Li, Wenjiang Huang and Yonghua Wang. Correlation Relationship between Satellite Remote Sensing Spectral Information and Eco-physiology Indexes of Winter Wheat at Flowering Period[J]. Journal of Triticeae Crops. 29(1), 79-82(2009).

[8] Xiaobo Zheng, Juan Chen, Weimin Kang, Yuxiang Luo and Pengju Tian. Methods of Monitoring Rice Growing Periods and Growth Status by Using MODIS in Yungui Plateau[J]. Chinese Journal of Agrometeorology. 28(4), 453-456,168(2007).

[9] Haboudane D, Miller J R, Tremblay N, et al. Integrated narrow-band vegetation indices for prediction of crop chlorophyll content for application to precision agriculture[J]. Remote Sensing of Environment. 81(2):416-426 (2002).

[10] Zhiding Guo, Zhihong Li, Xin Li, et al. Effects of Nitrogen Levels and the Methods on the Canopy NDVI, Nitrogen Content, Chlorophyll and Yield of Maize[J]. Journal of Maize Sciences. 21(6), 111-116(2013).

[11] Hu Yang. Changes of nitrogen distribution and rapid diagnosis for nitrogen nutrition status in the canopy leaf of rice[D]. Hangzhou Zhejiang, Zhejiang University. (2014).

[12] Jiping Jang. Dynamic characteristics of SPAD values of different positions leaves in the canopy of rice and its application for nitrogen nutrition[D]. Hangzhou Zhejiang, Zhejiang University. (2012).

[13] Jiping Jang, Jingping Yang, Zhengchao Yang, Junliang Zhou and Changshui Ge. Dynamic characteristics of SPAD value of rice leaf and adjacent leaf under different $\mathrm{N}$ application rates[J]. Journal of Zhejiang University(Agriculture and Life Sciences). 38(2), 166-174(2012).

[14] Junjun He. Effects of light and nitrogen levels on the distribution of canopy SPAD values and the diagnosis of nitrogen nutrition in rice leaf[D]. Hangzhou Zhejiang, Zhejiang University. (2014).

[15] Zhengjin Li, Weiguo Li, Shuanghe Shen. The Research of Object-oriented Classification Method in Remote Sensing Image with Texture Analysis-Taking Yangling County of Shanxi Province as an Example[J]. Remote Sensing Information. (8), 30-32(2009).

[16] Fenling Li, Li Wang, Jing Liu and Qingrui Chang. Remote Sensing Estimation of SPAD Value for Wheat Leaf Based on GF-1 Data[J]. Transactions of the Chinese Society for Agricultural Machinery. 25(3), 29-32(2005).

[17] Daughtry C S T, Walthall C L, Kim M S, et al. Estimating corn leaf chlorophyll concentration from leaf and canopy reflectance[J]. Remote Sensing of Environment. 74(2), 229-239(2000).

[18] Hunt E R, Daughtry C S T, Eitel J U H, et al. Remote sensing leaf chlorophyll content using a visible band index[J]. Agronomy Journal. 103(4), 1090-1099(2011).

[19] Fitzgerald G, Rodriguez D, O'Leary G. Measuring and predicting canopy nitrogen nutrition in wheat using a spectral index-The canopy chlorophyll content index (CCCI)[J].Field Crops Research. 116(3), 318-324(2011).

\section{Authors}

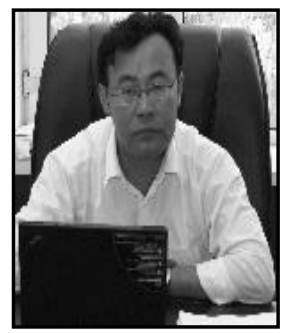

Zhongbin Su, (1965-), male, Ph.D., professor, president of the college of electrical and information in Northeast Agricultural University, mainly engaged in the research and application of information technology in agriculture. 